\title{
LEAFDATA: a literature-curated database for Arabidopsis leaf development
}

Dóra Szakonyi ${ }^{*}$

\begin{abstract}
Background: In the post-genomic era, biological databases provide an easy access to a wide variety of scientific data. The vast quantity of literature calls for curated databases where existing knowledge is carefully organized in order to aid novel discoveries. Leaves, the main photosynthetic organs are not only vital for plant growth but also essential for maintaining the global ecosystem by producing oxygen and food. Therefore, studying and understanding leaf formation and growth are key objectives in biology. Arabidopsis thaliana to this date remains the prime experimental model organism in plant science.

Description: LEAFDATA was created as an easily accessible and searchable web tool to assemble a relevant collection of Arabidopsis leaf literature. LEAFDATA currently contains 13,553 categorized statements from 380 processed publications. LEAFDATA can be searched for genes of interest using Arabidopsis Genome Initiative identifiers, for selected papers by means of PubMed IDs, authors and specific keywords. The results page contains details of the original publications, text fragments from the curated literature grouped according to information types and direct links to PubMed pages of the original papers.

Conclusions: The LEAFDATA database offers access to searchable entries curated from a large number of scientific publications. Due to the unprecedented details of annotations and the fact that LEAFDATA already provides records about approximately 1600 individual loci, this database is useful for the entire plant research community.
\end{abstract}

Keywords: Database, Arabidopsis thaliana, Leaf development, Manual literature curation

\section{Background}

Leaf development from primordium initiation to organ senescence is an intricate process controlled by interconnected regulatory pathways $[1,2]$. Many of the key genes have been thoroughly characterized, while the role of numerous other factors with clear leaf phenotypes has not been studied in the context of leaf organogenesis. The shoot apical meristem (SAM) gives rise to the aboveground differentiated organs. The position of leaf initiation is determined by polarized auxin accumulation generated by the YUCCA auxin biosynthesis genes [3] and the PIN-FORMED1 (PIN1) hormone transporter [4]. Leaf identity is established by suppression of meristem identity genes at this marked region by the MYB-family transcription factor ASYMMETRIC LEAVES1 (AS1) and

\footnotetext{
*Correspondence: dszakonyi@igc.gulbenkian.pt; info@leafdata.org
} Instituto Gulbenkian de Ciência, 2780-156 Oeiras, Portugal
$A S 2$, a LOB domain protein coding gene [5, 6]. A defined boundary region separates the meristem from the organ primordium and provides a border between neighboring organs. Organization of this domain depends on factors including CUP-SHAPED COTYLEDON (CUC) genes, LATERAL ORGAN BOUNDARIES (LOB), LATERAL ORGAN FUSION (LOF1), and JAGGED LATERAL ORGAN (JLO) genes [7]. The early leaf primordium emerges as radially symmetrical, cylindrical structure that soon differentiates along the proximodistal, mediolateral and dorsoventral axes. For the formation of a flattened leaf structure, mutually antagonistic developmental programs define the dorsal and ventral organ identity [8]. $A S 1, A S 2$ and the HD-ZIPIII genes act as ventral determinants, while the KANADI (KAN) genes, the $Y A B B Y$ genes and several AUXIN RESPONSE FACTORs (ARFs) promote ventral fate. Leaf growth is a coordinated process of cell division and cell expansion. Cell divisions are 
driven by a great number of cell cycle regulators such as cyclins, cyclin-dependent protein kinases, and inhibitors of cyclin-dependent kinases [9]. Many of these factors are also key players in DNA endoreduplication hence crucial for controlling cell size. Cell proliferation drives early stages of leaf development, while cell expansion dominates the later phases of leaf growth. During this process, pluripotent initial cells differentiate into the abaxial and adaxial epidermis, the palisade and spongy mesophyll cell layers and the vascular system. Specific genetic and molecular pathways drive the formation of guard cells [10] and trichomes [11]. Furthermore, analysis of mutant phenotypes revealed that genes involved in chromatin remodeling, pre-mRNA splicing and processing, protein translation, post-transcriptional regulation via small RNA pathways, proteasome-dependent protein degradation, hormonal signaling, metabolite biosynthesis and numerous other processes are essential for leaf organogenesis $[1,12-16]$.

During the past years, several public resources have been assembled focusing on Arabidopsis leaf development. Plant morphology depends on the combination of genetic determinants and environmental factors. Regular imaging and objective measurements are crucial to monitor quantitative traits. The PHENOPSIS DB [17] is built for data storage, sharing and analysis of the precise recordings of phenotypic variables and growth conditions from automated phenotyping platforms $[18,19]$. Additional measurements and offline microscopic analyses are manually added to each experiments. The database contains more than 93,000 plant images and 57,832 phenotypic details about 1057 Arabidopsis genotypes and offers data visualization and image analysis tools. The results of a systematic reverse genetic screen are summarized in PhenoLeaf $[20,21]$. Approximately 24,000 SALK mutant alleles were monitored for visible leaf defects. The 706 identified leaf mutants have been cataloged and can be queried by keywords for phenotype or genes. Another collection accommodating cleared leaves with visible vascular architecture including 412 Arabidopsis pictures are available in the ClearedLeavesDB [22, 23]. The leaf senescence database (LSD) focuses on the last phase of leaf development that leads to organ death [24-26]. Manual and computational data were collected about senescenceassociated genes (SAGs) from various plant species. The updated LSD 2.0 now contains 5356 genes and 322 mutants from 44 plant species, QTLs, seed information, sequence search functions and information about subcellular localization. Finally, the AGRON-OMICS consortium (Arabidopsis GROwth Network integrating OMICS technologies) was initiated to understand molecular mechanisms behind leaf growth using high-throughput experimental approaches. The effect of mild drought stress was studied in several stages of leaf development using transcript profiling and quantitative proteomics experiments [27]. These datasets along with metabolite measurements, photosynthesis and respiration rates, enzyme activities, ribosome numbers and lipid content are accessible at the project's data integration and data sharing portal [28]. In the framework of this project a novel literature curation method was developed using the Leaf Knowtator tool and 283 key publications were processed as a community effort [29]. It was demonstrated that the collected information could be integrated with other public resources and a relational database, KnownLeaf was created. Furthermore, a graphical network was built to facilitate knowledge mining. However, access to the curated is data is hindered by the lack of a web interface. Therefore, our main aim was to establish a convenient resource with reliable query functions for easy access to this curated library.

Here, we present LEAFDATA, a high-quality and freely available literature database for Arabidopsis leaf development. By searching and manually curating 380 primary research publications, we collected 13,553 statements about genes that were experimentally linked to leaf organogenesis. We have created LEAFDATA to support fundamental research and provide a solid information resource for our users.

\section{Construction and content \\ Data collection}

LEAFDATA records were collected by employing the customized Leaf Knowtator annotation tool [29]. This interface runs in Protégé software version 3.3.1 using and the Knowtator plug-in version 1.9 beta [30]. Result sections of full-text primary research papers are processed. Entries are collected into ten major categories: phenotype, gene expression, feature, DNA-protein interaction, protein-protein interaction, genetic interaction, process, regulation of gene expression, regulation of process, and regulation of phenotype (Table 1). All categories have predefined structures and information slots attached to them that can be filled with ontology terms already uploaded into Leaf Knowtator (Table 2). The main controlled ontology collections that are included in this project are Plant Ontology (PO) [31], BRENDA Tissue Ontology (BTO) [32], Phenotype, Attribute and Trait Ontology (PATO) [33], Plant Trait Ontology (TO) [34], Molecular Interaction (MI) [35], Plant Environment Ontology (EO) [36] and Gene Ontology (GO) [37]. Genes were associated with the specific AGI identifiers derived from the TAIR10 genome annotation [38]. In addition, the Knowtator plug-in automatically saves further details such as the annotated file, annotator and annotated text. The curation system is flexible and can be easily modified 
Table 1 Information types annotated in LEAFDATA

\begin{tabular}{|c|c|c|}
\hline Category & Example & Number \\
\hline Phenotype & The venation in each as 2 leaf lamina was bilaterally asymmetrical & 6559 \\
\hline Gene expression & YAB3 is detected in the abaxial regions of the developing leaves & 4617 \\
\hline Feature & the AP2/EREBP domain of LEP is located close to the N-terminus of the protein & 731 \\
\hline DNA-protein interactions & BES1 binding to the promoter of SAUR-15 & 151 \\
\hline Protein-protein interaction & AtCul1 ... co-immunoprecipitated with ... myc-tagged ASK1 & 345 \\
\hline Genetic interaction & se quantitatively and qualitatively enhanced the lobing of as $1 . .$. leaves & 382 \\
\hline Process & CYCD3;1 ... important for the initiation of cell division at the $\mathrm{G} 1$ phase in leaves & 348 \\
\hline Regulation of gene expression & we conclude that STM negatively regulates AS1 & 171 \\
\hline Regulation of process & WRKY53, is an important positive regulator of senescence & 206 \\
\hline Regulation of phenotype & NEK6 ... promotes biomass levels & 42 \\
\hline
\end{tabular}

Ten major classes of information are curated in our database. Examples of these categories and number of statements are shown here

to other annotation projects. Required slots are filled with terms closely following the original text. In addition to the community curations from 283 publications from the AGRON-OMICS project, 97 new papers were processed.

\section{Database construction}

Annotations were exported as XML files from Leaf Knowtator. These files are small and easy to share. The XML files were transformed into a single table with a custom made Perl script [24] and loaded in bulk using Structured Query Language (SQL) queries. The LEAFDATA Database resides on the MS SQL Server 2008 platform. The website design is fully responsive in line with current industry standards and is based on the Bootstrap Framework. Bootstrap utilizes HTML, CSS, and JS frameworks for developing responsive projects on the web. For database integration the server side engine Adobe Coldfusion 9 running over MS IIS was chosen for its relatively inexpensive hosting costs, its rapid development credentials and powerful data collation functions. The employment of these cutting-edge technologies offers a modern, literature-curated website that can be used on any device and provide fast access to our data in any research environment.

\section{Utility}

\section{LEAFDATA home}

The main site (Fig. 1) provides direct access to the search functions. There is a visual representation of the database content including number of curated publications and individual statements, and details of the different categories. Upon selecting any categories, all annotations can be retrieved. On the bottom of the page, a news section can be found directly connected to an active Twitter account with announcements of relevant publications and database updates.
Moreover, a direct contact form is available for any enquiries.

\section{LEAFDATA search tools}

LEAFDATA provides four convenient search functions. Genes of interest can be queried by using unique AGI identifiers based on the last TAIR10 genome release. All annotations can be retrieved from a selected publication using the PubMed ID. In addition to an author query, we also offer a keyword search. Results are arranged according to distinct categories and individual publications. For illustration, records from an AGI search for the $H D$ ZIPIII transcription factor REVOLUTA (REV) is shown (Fig. 2; Table 3). This query resulted in 78 statements from 17 different papers. The keyword tool is particularly helpful to attain required information. It allows combining multiple keywords and limits the search results to only those documents that contain all the terms. This function can be used effectively to find plant lines that share a certain phenotype, genes with the same biological function or similar expression domains. Recent publications revealed that genetic combinations of plant lines with increased leaf size can further enhance growth [39]. In order to find all the large-leaf Arabidopsis lines curated in LEAFDATA, we performed a search for the terms size_PATO:0000586 and increased size_PATO:0000117 and retrieved a preliminary list of 173 statements (Additional file 1). Ontology terms were used to minimize the recovery of false positive records and 'plant part' was not specified to maximize the number of genuine hits. Terms with similar meanings can be used for this query. For example, large leaves, big leaves, increased leaf size gave 162, 12, and 373 results, respectively (Additional file 2: Table S1). Ten statements were randomly selected for additional data mining (Table 4). First AGI codes were collected from the LEAFDATA gene list available under the SEARCH LEAFDATA tab (see also Additional File 
Table 2 Phenotype annotation exported from Leaf Knowtator

\begin{tabular}{|c|c|}
\hline Database columns & Entries \\
\hline File & 21401745.txt.knowtator.xml \\
\hline Class & Phenotype \\
\hline Annotator & Dora Szakonyi, LEAFDATA \\
\hline Spanned text & $\begin{array}{l}\text { The rid2-1 mutant was temperature sensitive for } \\
\text { seedling growth as well as for callus formation. } \\
\text { In rid2-1 seedlings grown at } 22^{\circ} \mathrm{C} \text {, the true } \\
\text { leaves were pointed }\end{array}$ \\
\hline Annotated text & $\begin{array}{l}\text { Genotype } I D=\text { rid2- } 1 \text { Property Slot }=\text { NULL|Value } \\
I D=\text { pointed } \mid \text { Plant part } I D=\text { leaves }\end{array}$ \\
\hline \multicolumn{2}{|l|}{ Growth condition } \\
\hline \multicolumn{2}{|l|}{ Developmental stage } \\
\hline \multicolumn{2}{|l|}{ Localisation } \\
\hline Property & shape_PATO:0000052 \\
\hline \multicolumn{2}{|l|}{ Process } \\
\hline Value & pointed_PATO:0002258 \\
\hline \multicolumn{2}{|l|}{ Regulation } \\
\hline \multicolumn{2}{|l|}{ Gene expression } \\
\hline \multicolumn{2}{|l|}{ Gene studied } \\
\hline \multicolumn{2}{|l|}{ Interaction type } \\
\hline \multicolumn{2}{|l|}{ Protein studied } \\
\hline \multicolumn{2}{|l|}{ Interactor protein } \\
\hline \multicolumn{2}{|l|}{ Protein } \\
\hline \multicolumn{2}{|l|}{ Gene target } \\
\hline \multicolumn{2}{|l|}{ Genetic interactor } \\
\hline \multicolumn{2}{|l|}{ DNA target } \\
\hline Genotype ID & mutated gene_MI:0804 \\
\hline Genotype details & $\begin{array}{l}\text { Gene ID = AT5G57280 | Genotype_Zygo- } \\
\text { sity= homozygous diploid_APO:0000229 } \\
\text { | Mutant LOF_GOF ID = loss of function_ } \\
\text { APO:0000011 }\end{array}$ \\
\hline Factuality & \\
\hline
\end{tabular}

In LEAFDATA, a complex range of information is attached to each displayed statements deposited as spanned text. Different classes have distinct predefined slots however records are converted into a single table. Database columns corresponds to all available Leaf Knowtator slots. File, Annotator, Spanned text and Annotated text information is automatically added to curated statements

3: Table S2) then AGI searches were performed for the individual genes. Further analysis was focused on gene expression data in wild-type background and reported biological functions. For eight genes, both gene expression and functional records were recovered. In one case, only gene expression data was found while for a sole example none of the required additional information was available in LEAFDATA. Importantly, half of these records were gathered from multiple (2-4) papers.

All the query tools can be accessed from the main site as well as from dedicated search pages where queries can be restricted to different categories. Finally, to show the full content of LEAFDATA, there is a current list of all annotated papers under the SEARCH LEAFDATA tab (Additional File 4: Table S3).

\section{Discussion}

Leaves are essential organs for plant life and the location of multiple biological processes. Organogenesis from emergence of leaf primordium through pattern formation, maturation, maintenance until senescence is regulated by diverse regulatory pathways. Genetic and molecular roles of numerous genes were described in great detail. These genes are classified as key players in leaf morphogenesis. However, numerous additional genes causing altered leaf morphology have been isolated. In many cases, characterization of the observed leaf phenotypes are not main scope of these studies. Furthermore, these information are scattered throughout the existing scientific literature. Our aim was to create a convenient public collection of relevant leaf literature that provides simple query functions and easy access to a large library at the same time. Here, we demonstrate that our published annotation method and the Leaf Knowtator interface [29] can be used effectively for establishing high-quality literature resources. Employing this system guaranteed several unique database features. With a quick workflow, we are able to retain a large amount of information. In LEAFDATA, not only are the curated text fragments from the original publications kept and displayed but ontology terms from established structured vocabularies are simultaneously attached to these statements. Using these standardized terms helps building complex queries and can facilitate data sharing and integration [40]. We adhere to further community standards by employing the entity-attribute-value (EAV) model for phenotype annotations [41]. On average, more than 35 annotations per publication are generated adding up to a total of 13,553 independent statements about nearly 1300 genes. A major advantage of our database is that our curations are not restricted to single genotypes or information types. For instance, phenotype annotations can cover descriptions of single and multiple mutants (Table 3) as well as constitutive or inducible overexpressors, transgenic plants expressing chimeric constructs or modified versions of the gene of interest. Also, gene expression records provide an exceptional range of information including quantification of expression levels and spatial distribution in wild type or various mutant backgrounds (Table 3). Most of our annotations belong to the phenotype and gene expression class however numerous protein-protein interaction, genetic interaction and DNA-protein interaction records can be accessed (Table 1). The original publication details (author, title, PubMed ID) are clearly displayed with each statements and a direct link is provided to the dedicated PubMed 


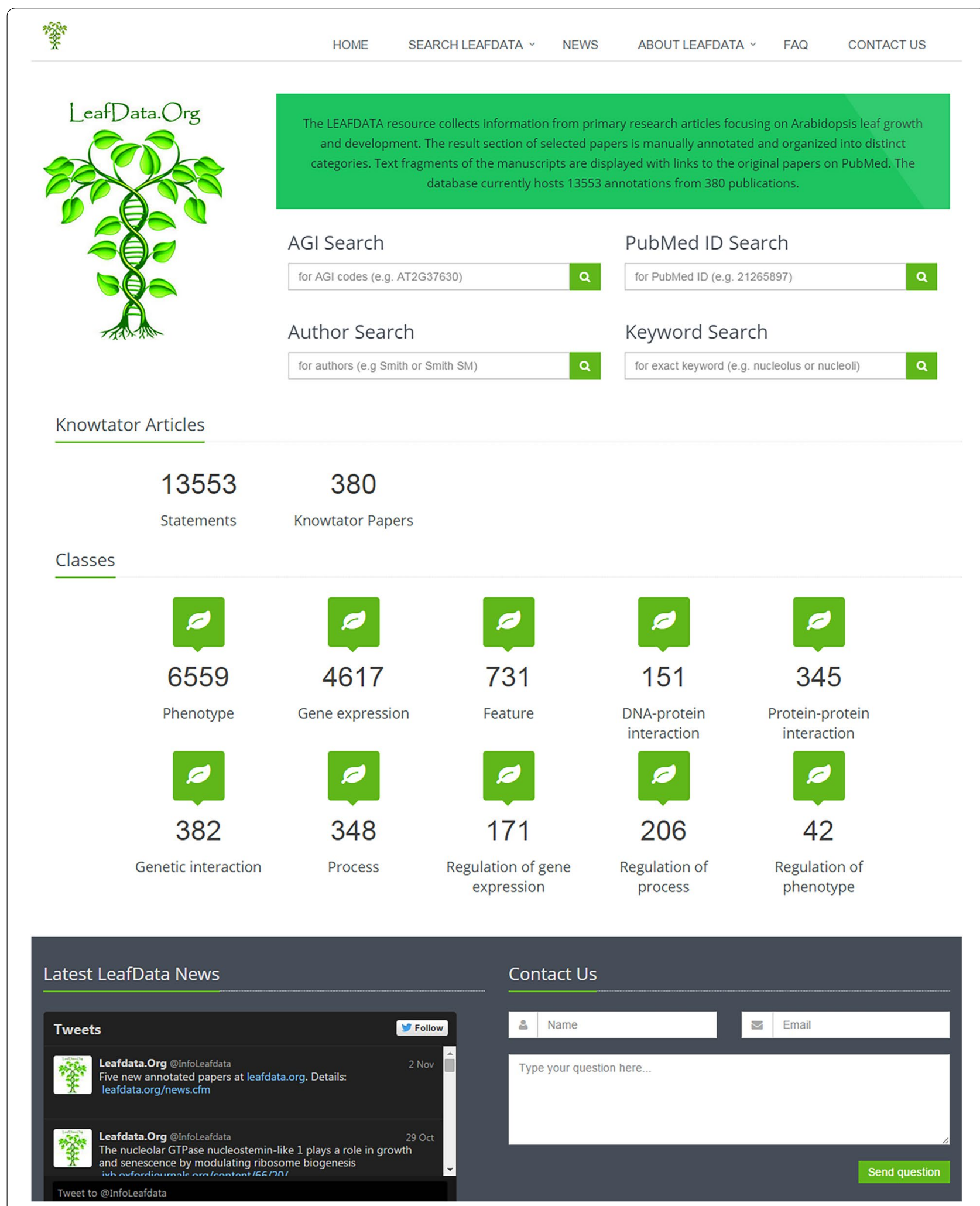

Fig. 1 LEAFDATA home. Four key search tools, a summary of the database content, a news section connected to an active Twitter account and a contact form can be reached from the LEAFDATA main page 


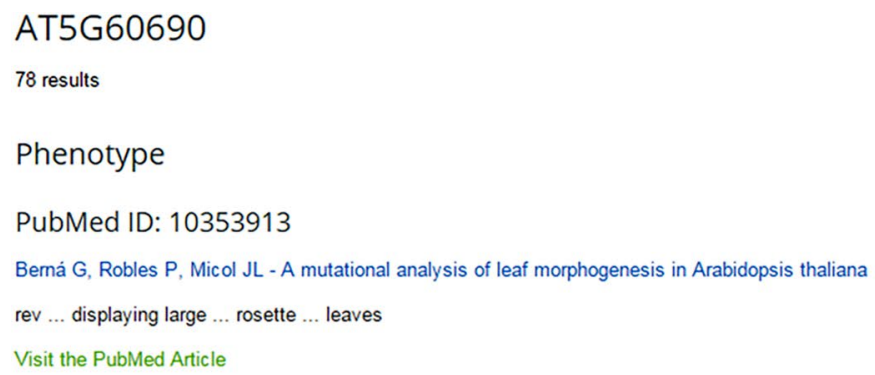

Fig. 2 LEAFDATA result page. AGI search for AT5G60690 was performed. Records are organized according to information types and publications with direct links to the PubMed collection

page. The search functions were designed to give a quick access to records from a chosen gene, paper or author. The keyword query allows more detailed data mining e.g. for a specific genotype using multiple terms. In summary, the combination of the LEAFDATA tools can be used effectively to collect wide-range of information (Table 4).
LEAFDATA is a useful platform not only for researchers interested in leaf development but for scientists working with other traits, plant species or model organisms. There are possible applications for our dataset in large-scale projects, mutagenesis screens and developing text-mining tools. University students, interested professionals and the general public can benefit from free and 
Table 3 Results using AGI search for AT5G60690

Phenotype

PubMed ID: 10353913

Berná G, Robles P, Micol JL_A mutational analysis of leaf morphogenesis in Arabidopsis thaliana

rev ... displaying large ... rosette ... leaves

PubMed ID: 16284709

Horiguchi G, Ferjani A, Fujikura U, Tsukaya H-Coordination of cell proliferation and cell expansion in the control of leaf size in Arabidopsis thaliana known large-leaf mutants ... rev

PubMed ID: 20807212

Kim HS, Kim SJ, Abbasi N, Bressan RA, Yun DJ, Yoo SD, Kwon SY, Choi SB - The DOF transcription factor Dof5.1 influences leaf axial patterning by promoting Revoluta transcription in Arabidopsis

the 35S::REV Arabidopsis plant displayed an upwardly curled leaf phenotype (Mallory et al. 2004)

PubMed ID: 22003085

Wang W, Xu B, Wang H, Li J, Huang H, Xu L_-YUCCA genes are expressed in response to leaf adaxial-abaxial juxtaposition and are required for leaf margin development

as 2-1 rev-1 ... there were many more margin cell patches than in the wild type

as2-1 rev-1 yuc1 yuc2 yuc4 ... The margin formation ... was severely defective

as2-1 rev-1 ... leaves ... had fewer hydathodes than the wild type

leaves of ... as 2-1 rev-1 ... were smaller than fully expanded leaves of the wild type

finger-shaped protrusions ... were observed on the adaxial side of late-appearing leaves of as 2-1 rev-1

as2-1 rev-1 ... cell patches ... could occur anywhere among the margin cells

The rosette leaves of one as2-1 rev-1 yuc1 yuc2 yuc4 plant ... leaf margin curled upward

as2-1 rev-1 yuc1 yuc2 yuc4 ... leaves, which were filamentous

treatment with increased NPA concentrations resulted in decreased plant size of as2-1 rev-1

as $2-1$ rev-1 ... had lobes on their rosette leaves

Compared with the as2-1 rev-1 double mutant ... as2-1 rev-1 yuc1 yuc2 yuc4/+ plants were reduced in size

The rosette leaves of one as2-1 rev-1 yuc1 yuc2 yuc4 plant ... were narrow

as2-1 rev-1 yuc1 yuc2 yuc4 ... lacking typical margin cells

PubMed ID: 7555701

Talbert PB, Adler HT, Parks DW, Comai L-The REVOLUTA gene is necessary for apical meristem development and for limiting cell divisions in the leaves and stems of Arabidopsis thaliana

leaves of rev-1 mutants grew abnormally large

The rosette leaves of rev-1 plants were not readily distinguishable from wild-type No-0 leaves prior to bolting. As bolting began, however, the youngest rosette leaves became abnormally large ... as they matured

The rev-1 mutation caused overgrowth of both rosette and cauline leaves

The rosette leaves of rev-1 plants were not readily distinguishable from wild-type No-0 leaves prior to bolting. As bolting began, however, the youngest rosette leaves became ... distorted or uneven in shape as they matured

mutants with a syndrome con- sisting of revolute (downwardly curled) leaves ... rev-1, rev-2 and rev-4

Both the leaves and the primary shoots of rev-1 mutants were often darker green than those of wild type

The difference in leaf size between wild-type and rev-1 plants was not obvious in the earlier rosette leaves, but we measured significant size differences in the cotyledons and first and third leaves from cohort 3 (Table 2). Later leaves differed more dramatically: the mean length of the longest rosette leaf (ordinarily the youngest leaf) of rev-1 plants was about $39 \%$ longer than wild-type controls, and rev-1 cauline leaves became up to twice as long as their wild-type counterparts

Gene expression

PubMed ID: 11525739

Eshed Y, Baum SF, Perea JV, Bowman JL-Establishment of polarity in lateral organs of plants

mRNA of REV ... Later expression is confined to the provascular and vascular tissues of leaves

mRNA of REV ... restricted to the adaxial domain as developing primordia

REV ... initiates normally in kan1 kan2 leaf primordia

MRNA of REV ... is localized to the SAM

REV ... in kan1 kan2 leaf primordia ... confinement to the adaxial domain is delayed

mRNA of REV ... is localized to the SAM, throughout leaf primordia anlagen

PubMed ID: 12615938

Nelissen H, Clarke JH, De Block M, De Block S, Vanderhaeghen R, Zielinski RE, Dyer T, Lust S, Inzé D, Van Lijsebettens M-DRL1, a homolog of the yeast TOT4/KTI12 protein, has a function in meristem activity and organ growth in plants

pREV(OLUTA)-GUS ... were introgressed into drl1-2 ... marker lines displayed promoter activity in the dorsal part of the leaf primordium, including the vascular bundles

PubMed ID: 16682355

Garcia D, Collier SA, Byrne ME, Martienssen RA-Specification of leaf polarity in Arabidopsis via the trans-acting siRNA pathway

REVOLUTA ... Expression is re- stricted to the adaxial domain 
Table 3 continued

PubMed ID: 16699177

Xu L, Yang L, Pi L, Liu Q, Ling Q, Wang H, Poethig RS, Huang H_-Genetic interaction between the AS1-AS2 and RDR6-SGS3-AGO7 pathways for leaf morphogenesis

leaves of the sgs3-11 as2-101 ... contained ... reduced levels of REV transcripts

REV transcripts were detected in the earlier stage leaf primordia in ... zip as 1-101

leaves of the ... zip as2-101 ... contained ... reduced levels of REV transcripts

REV ... repressed ... in the rdr6-3 as2-101 leaves

REV transcripts were detected in the earlier stage leaf primordia in ... sgs3-11 as2-101

rdr6-3 as2-101 ... leaves ... contained ... reduced levels of REV transcripts

REV transcripts were detected in the earlier stage leaf primordia in ... zip as2-101

REV transcripts were detected in the earlier stage leaf primordia in sgs3-11 as 1-101

PubMed ID: 17559509

Iwakawa H, Iwasaki M, Kojima S, Ueno Y, Soma T, Tanaka H, Semiarti E, Machida Y, Machida C—Expression of the ASYMMETRIC LEAVES2 gene in the adaxial domain of Arabidopsis leaves represses cell proliferation in this domain and is critical for the development of properly expanded leaves

in as $2-1$... No significant differences in levels of transcripts were detected for ... REV

in as 1-1 ... No significant differences in levels of transcripts were detected for ... REV

PubMed ID: 19717616

Vandenbussche M, Horstman A, Zethof J, Koes R, Rijpkema AS, Gerats T—Differential recruitment of WOX transcription factors for lateral development and organ fusion in Petunia and Arabidopsis

In Arabidopsis, organ polarity regulation has been characterized extensively at the molecular level. We therefore have monitored the expression levels of a selection of Arabidopsis genes known to be involved in abaxial/adaxial patterning. The selection of genes comprises ... REVOLUTA ... None of the monitored Arabidopsis genes exhibited significant changes in transcript levels between wox 1 prs mutant samples and the wild type

PubMed ID: 20628155

Sarojam R, Sappl PG, Goldshmidt A, Efroni I, Floyd SK, Eshed Y, Bowman JL—Differentiating Arabidopsis shoots from leaves by combined YABBY activities YABBY triple mutants display reduced expression of ... REV

PubMed ID: 20807212

Kim HS, Kim SJ, Abbasi N, Bressan RA, Yun DJ, Yoo SD, Kwon SY, Choi SB-The DOF transcription factor Dof5.1 influences leaf axial patterning by promoting Revoluta transcription in Arabidopsis

REV and ATHB-15 trans-cripts were strongly enhanced in Dof5.1-D (Figure 6a); however, ATHB-15 was not increased in DEX::Dof5.1 plants upon DEX treatment whereas REV transcript was enhanced (Figure 6b). Increased expression of ATHB-15 in Dof5.1-D is probably due to a secondary effect REV expression was decreased in 35S::Dof5.1 $\triangle$ Act plants although the levels were different depending on transgenic lines

PubMed ID: 21223391

Szakonyi D, Byrne ME-Ribosomal protein L27a is required for growth and patterning in Arabidopsis thaliana

REV:REV-VENUS ... expressed in the apical and central regions of wild-type embryos

PubMed ID: 21251100

Horiguchi G, Mollá-Morales A, Pérez-Pérez JM, Kojima K, Robles P, Ponce MR, Micol JL, Tsukaya H—Differential contributions of ribosomal protein genes to Arabidopsis thaliana leaf development

agreement with the synergistic polarity defects observed in rpl4d-3 as2-1 ... expression ... REV were expressed at similar levels in the ... parents

PubMed ID: 22003085

Wang W, Xu B, Wang H, Li J, Huang H, Xu L_Y YUCCA genes are expressed in response to leaf adaxial-abaxial juxtaposition and are required for leaf margin development

pDR5::GUS ... staining in the as2-1 rev-1 ... leaves was also concentrated in the top portion of the protrusions

YUC4 was expressed in the leaf protrusions of the mock-treated as 2-1 rev-1

as $2-1$ rev-1 ... when ... treated with 1 MM NPA ... we did not observe the small GUS-staining spots on leaf surfaces

as $2-1$ rev-1 ... expressions of ... YUC4 ... associated with the protrusions on leaves

as $2-1$ rev-1 ... expressions of YUC1 ... associated with the protrusions on leaves

as $2-1$ rev-1 ... expressions of ... YUC2 ... associated with the protrusions on leaves

PubMed ID: 22026817

Xu D, Huang W, Li Y, Wang H, Huang H, Cui X-Elongator complex is critical for cell cycle progression and leaf patterning in Arabidopsis

We next examined expression of leaf polarity marker genes REVOLUTA (REV) and FILAMENTOUS FLOWER (FIL) in the elo2 as 2 background. rev-9 is a

T-DNA enhancer trap line in which $\beta$-glucuronidase (GUS) staining represents the expression of the leaf adaxial marker REV (Emery et al. 2003; Hawker and Bowman 2004). Compared with that in the rev-9/+ plant (Fig. 1q), GUS staining was not detected from needle-like leaves of the elo2 as2 rev-9/+ plant

PubMed ID: 23268445

Ben Chaabane S, Liu R, Chinnusamy V, Kwon Y, Park JH, Kim SY, Zhu JK, Yang SW, Lee BH._STA1, an Arabidopsis pre-mRNA processing factor 6 homolog, is a new player involved in miRNA biogenesis

Compared with WT, the accumulation of ... REV transcripts was higher in sta1-1, which is linked to decreased miR164/166 levels and explains the serrated leaf phenotype of sta1-1 
Table 3 continued

PubMed ID: 24464295

Huang T, Harrar Y, Lin C, Reinhart B, Newell NR, Talavera-Rauh F, Hokin SA, Barton MK, Kerstetter RA—Arabidopsis KANADI1 acts as a transcriptional repressor by interacting with a specific cis-element and regulates auxin biosynthesis, transport, and signaling in opposition to HD-ZIPIII factors we found evidence for regulation by ... GR-REV of ... At5g47800

A third technique, qRT-PCR, on independent samples confirmed statistically significant upregulation of NPY1 by GR-REV in the presence and absence of CHX, indicating that NPY1 is likely a direct target of REV activation

qRT-PCR on an independent set of samples showed upregulation of WAG1 by GR-REV both in the presence and absence of CHX

we found evidence for regulation by ... GR-REV of ... ARF3

we found evidence for regulation by ... GR-REV of ... LAX3

we found evidence for regulation by ... GR-REV of ... YUCCA5

we found evidence for regulation by ... GR-REV of ... LAX1

we found evidence for regulation by ... GR-REV of ... At1g50280

we found evidence for regulation by ... GR-REV of ... LAX2

we found evidence for regulation by ... GR-REV of ... TAA1

we found evidence for regulation by ... GR-REV of ... ENP1/NPY1

ARF3/ETTIN showed reproducible upregulation by GR-REV

we found evidence for regulation by ... GR-REV of ... At1g52770

DNA-protein interaction

PubMed ID: 20807212

Kim HS, Kim SJ, Abbasi N, Bressan RA, Yun DJ, Yoo SD, Kwon SY, Choi SB-The DOF transcription factor Dof5.1 influences leaf axial patterning by promoting Revoluta transcription in Arabidopsis

The results from both in vitro and in vivo binding assays demonstrate that Dof5.1 directly binds to the REV promoter

The EMSA result showed that, GST alone did not bind to the 89-bp long substrate (Figure 6d left, lane 2) but the GST-Dof5.1DB fusion protein migrated with promoter DNA

Genetic interaction

PubMed ID: 20807212

Kim HS, Kim SJ, Abbasi N, Bressan RA, Yun DJ, Yoo SD, Kwon SY, Choi SB-The DOF transcription factor Dof5.1 influences leaf axial patterning by promoting Revoluta transcription in Arabidopsis

The resulting Dof5.1-D/rev plants lacked the upward-curling phenotype of Dof5.1-D, thereby displaying almost WT morphology

PubMed ID: 22003085

Wang W, Xu B, Wang H, Li J, Huang H, Xu L-YUCCA genes are expressed in response to leaf adaxial-abaxial juxtaposition and are required for leaf margin development

leaves ... as 2-1 rev-1 ... were smaller than ... those of the corresponding single mutants

Process

PubMed ID: 16682355

Garcia D, Collier SA, Byrne ME, Martienssen RA—Specification of leaf polarity in Arabidopsis via the trans-acting siRNA pathway

REVOLUTA ... specifying adaxial identity

Regulation of gene expression

PubMed ID: 16682355

Garcia D, Collier SA, Byrne ME, Martienssen RA—Specification of leaf polarity in Arabidopsis via the trans-acting siRNA pathway

The microRNA miR165, which regulates class III HD- ZIP gene expression through transcript cleavage

REVOLUTA ... Expression is re- stricted ... by KANADI (KAN) genes

PubMed ID: 20807212

Kim HS, Kim SJ, Abbasi N, Bressan RA, Yun DJ, Yoo SD, Kwon SY, Choi SB - The DOF transcription factor Dof5.1 influences leaf axial patterning by promoting Revoluta transcription in Arabidopsis

These results strongly indicate that Dof5.1 activates REV transcription

PubMed ID: 24464295

Huang T, Harrar Y, Lin C, Reinhart B, Newell NR, Talavera-Rauh F, Hokin SA, Barton MK, Kerstetter RA-Arabidopsis KANADI1 acts as a transcriptional repressor by interacting with a specific cis-element and regulates auxin biosynthesis, transport, and signaling in opposition to HD-ZIPIII factors

REV increases transcription, most likely by direct activation, of NPY1

Regulation of phenotype

PubMed ID: 16682355

Garcia D, Collier SA, Byrne ME, Martienssen RA—Specification of leaf polarity in Arabidopsis via the trans-acting siRNA pathway

REVOLUTA, influence leaf shape

Seventy-eight records are available in LEAFDATA for AT5G60690, REV gene. These statements are organized according to information type and original publication

easy access to the LEAFDATA library offering processed scientific records.

We envision future improvements for LEAFDATA. The current database contains approximately $15-20 \%$ of the published Arabidopsis leaf literature, is constantly being updated. However, it will take significant effort to annotate every existing leaf development paper and at the same time keep up with the steady flow of new research. We plan to develop advanced search functions for instance queries for specific phenotypic characteristics, 


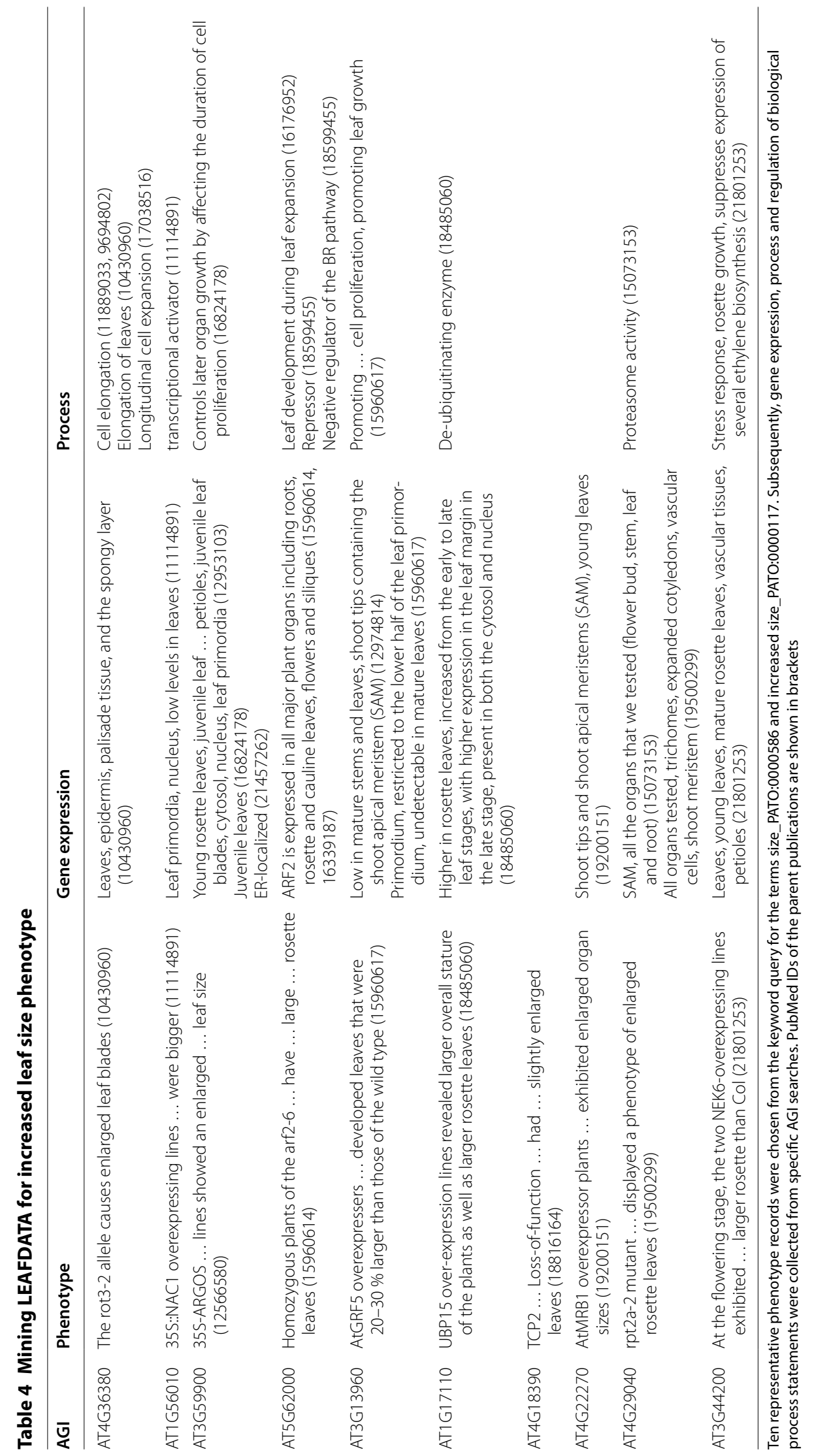


combinations of features or exclusion certain traits. Similarly, gene expression statements can be further explored by genotypes, changes in certain target genes or expression in special subcellular compartments, cell types and organs. Lastly, we are interested in data visualization and integration with other datasets.

\section{Conclusions}

The sheer amount of scientific literature is calling for carefully curated database summarizing experimental results. We employed the Leaf Knowtator curation system and constructed a unique, comprehensive database focusing on Arabidopsis leaf development. In addition to previously described regulators, genes with clear leaf phenotypes are included. The LEAFDATA collection gives access to 380 publications organized according to papers and information types. Four query functions provide easy access to high-quality annotations and direct links to the original papers. LEAFDATA serves as a valuable resource and reference point for the research community. Finally, our annotation approach, data organization and database structure can serve as a prototype for other literature curation projects.

\section{Availability and requirements}

LEAFDATA is an open access database at www.leafdata. org. The collection is updated on a regular basis. Questions, comments and requests regarding this database should be sent to Dóra Szakonyi at info@leafdata.org.

Details of LEAFDATA content and screenshots were recorded on $08 / 11 / 2015$.

\section{Additional files}

Additional file 1. Results using keyword search for size_PATO:0000586 increased size_PATO:0000117. A preliminary list of 173 results were recovered. Confirmed records are highlighted in yellow.

Additional file 2: Table S1. Number of results retrieved by keywords search using different terms for large-leaf phenotype.

Additional File 3: Table S2. List of genes curated in LEAFDATA.

Additional File 4: Table S3. List of papers curated in LEAFDATA.

\section{Acknowledgements}

We would like to acknowledge Ross Austen at Dataweaver International for all the help with the webpage creation. We thank Sofie Van Landeghem and Dean Ravenscroft for feedback on the LEAFDATA resource. We are grateful for Amanda Rasmussen, Ken W. Berendzen and Gerda Szakonyi for useful comments on the database and the manuscript. This work was conducted using the Protégé resource, which is supported by Grant GM10331601 from the National Institute of General Medical Sciences of the United States National Institutes of Health.

\section{Competing interests}

The author declares that he has no competing interests.
Received: 1 December 2015 Accepted: 8 February 2016

Published online: 15 February 2016

\section{References}

1. Kalve S, De Vos D, Beemster GT. Leaf development: a cellular perspective. Front Plant Sci. 2014;5:362

2. Rodriguez RE, Debernardi JM, Palatnik JF. Morphogenesis of simple leaves: regulation of leaf size and shape. Wiley Interdiscip Rev Dev Biol. 2014;3(1):41-57.

3. Cheng Y, Dai X, Zhao Y. Auxin synthesized by the YUCCA flavin monooxygenases is essential for embryogenesis and leaf formation in Arabidopsis. Plant Cell. 2007;19(8):2430-9.

4. Reinhardt D, Pesce ER, Stieger P, Mandel T, Baltensperger K, Bennett $\mathrm{M}$, et al. Regulation of phyllotaxis by polar auxin transport. Nature. 2003;426(6964):255-60.

5. Byrne ME, Barley R, Curtis M, Arroyo JM, Dunham M, Hudson A, Martienssen RA. Asymmetric leaves1 mediates leaf patterning and stem cell function in Arabidopsis. Nature. 2000;408(6815):967-71.

6. Semiarti E, Ueno Y, Tsukaya H, Iwakawa H, Machida C, Machida Y. The ASYMMETRIC LEAVES2 gene of Arabidopsis thaliana regulates formation of a symmetric lamina, establishment of venation and repression of meristem-related homeobox genes in leaves. Development. 2001;128(10):1771-83.

7. Zadnikova P, Simon R. How boundaries control plant development. Curr Opin Plant Biol. 2014;17:116-25.

8. Szakonyi D, Moschopoulos A, Byrne ME. Perspectives on leaf dorsoventral polarity. J Plant Res. 2010;123(3):281-90.

9. Blomme J, Inze D, Gonzalez N. The cell-cycle interactome: a source of growth regulators? J Exp Bot. 2014;65(10):2715-30.

10. Lau OS, Bergmann DC. Stomatal development: a plant's perspective on cell polarity, cell fate transitions and intercellular communication. Development. 2012;139(20):3683-92.

11. Pattanaik S, Patra B, Singh SK, Yuan L. An overview of the gene regulatory network controlling trichome development in the model plant, Arabidopsis. Front Plant Sci. 2014;5:259.

12. Gentry M, Hennig L. Remodelling chromatin to shape development of plants. Exp Cell Res. 2014;321(1):40-6.

13. Tsukaya H, Byrne ME, Horiguchi G, Sugiyama M, Van Lijsebettens M, Lenhard M. How do 'housekeeping' genes control organogenesis?Unexpected new findings on the role of housekeeping genes in cell and organ differentiation. J Plant Res. 2013;126(1):3-15.

14. Kidner CA. The many roles of small RNAs in leaf development. J Genet Genomics. 2010;37(1):13-21.

15. Kurepin LV, Pharis RP. Light signaling and the phytohormonal regulation of shoot growth. Plant Sci. 2014;229:280-9.

16. Perez-Perez JM, Candela H, Robles P, Quesada V, Ponce MR, Micol JL. Lessons from a search for leaf mutants in Arabidopsis thaliana. Int J Dev Biol. 2009;53(8-10):1623-34.

17. PHENOPSIS DB. http://bioweb.supagro.inra.fr/phenopsis/. Accessed 08 Nov 2015.

18. Fabre J, Dauzat M, Negre V, Wuyts N, Tireau A, Gennari E, et al. PHENOPSIS DB: an information system for Arabidopsis thaliana phenotypic data in an environmental context. BMC Plant Biol. 2011;11:77.

19. Granier C, Aguirrezabal L, Chenu K, Cookson SJ, Dauzat M, Hamard P, et al. PHENOPSIS, an automated platform for reproducible phenotyping of plant responses to soil water deficit in Arabidopsis thaliana permitted the identification of an accession with low sensitivity to soil water deficit. New Phytol. 2006;169(3):623-35.

20. Wilson-Sanchez D, Rubio-Diaz S, Munoz-Viana R, Perez-Perez JM, Jover-Gil S, Ponce MR, et al. Leaf phenomics: a systematic reverse genetic screen for Arabidopsis leaf mutants. Plant J. 2014;79(5):878-91.

21. PhenoLeaf. http://genetics.umh.es/phenoleaf/index.php. Accessed 08 Nov 2015.

22. Das A, Bucksch A, Price CA, Weitz JS. ClearedLeavesDB: an online database of cleared plant leaf images. Plant Methods. 2014;10(1):8.

23. ClearedLeavesDB. http://clearedleavesdb.org/. Accessed 08 Nov 2015.

24. Liu X, Li Z, Jiang Z, Zhao Y, Peng J, Jin J, et al. LSD: a leaf senescence database. Nucl Acids Res. 2011;39(Database issue):D1103-7. 
25. Li Z, Zhao Y, Liu X, Peng J, Guo H, Luo J. LSD 2.0: an update of the leaf senescence database. Nucl Acids Res. 2014;42:D1200-5.

26. Leaf Senescence Database. http://www.eplantsenescence.org/. Accessed 08 Nov 2015

27. Baerenfaller K, Massonnet C, Walsh S, Baginsky S, Buhlmann P, Hennig L, et al. Systems-based analysis of Arabidopsis leaf growth reveals adaptation to water deficit. Mol Syst Biol. 2012;8:606.

28. AGRON-OMICS data integration and data sharing portal. https://agronomics.ethz.ch/. Accessed 08 Nov 2015.

29. Szakonyi D, Van Landeghem S, Baerenfaller K, Baeyens L, Blomme J, Casanova-Sáez R, et al. The KnownLeaf literature curation system captures knowledge about Arabidopsis leaf growth and development and facilitates integrated data mining. Curr Plant Biol. 2015;2015(2):1-11.

30. Ogren PV. Knowtator: a protégé plug-in for annotated corpus construction. In: Proceedings of the 2006 Conference of the North American Chapter of the Association for Computational Linguistics on Human Language Technology: companion volume: demonstrations; New York, New York. 1225791: Association for Computational Linguistics; 2006. p. 273-275.

31. Jaiswal P, Avraham S, Ilic K, Kellogg EA, McCouch S, Pujar A, et al. Plant ontology (PO): a controlled vocabulary of plant structures and growth stages. Comp Funct Genomics. 2005;6(7-8):388-97.

32. Gremse M, Chang A, Schomburg I, Grote A, Scheer M, Ebeling C, et al. The BRENDA tissue ontology (BTO): the first all-integrating ontology of all organisms for enzyme sources. Nucl Acids Res. 2011;39(Database issue):D507-13.
33. Gkoutos GV, Green EC, Mallon AM, Hancock JM, Davidson D. Using ontologies to describe mouse phenotypes. Genome Biol. 2005;6(1):R8.

34. Jaiswal P, Ware D, Ni J, Chang K, Zhao W, Schmidt S, et al. Gramene: development and integration of trait and gene ontologies for rice. Comp Funct Genomics. 2002;3(2):132-6.

35. Hermjakob H, Montecchi-Palazzi L, Bader G, Wojcik J, Salwinski L, Ceol A et al. The HUPO PSI's molecular interaction format-a community standard for the representation of protein interaction data. Nat Biotechnol. 2004;22(2):177-83.

36. Youens-Clark K, Buckler E, Casstevens T, Chen C, Declerck G, Derwent P, et al. Gramene database in 2010: updates and extensions. Nucl Acids Res. 2011;39(Database issue):D1085-94.

37. Ashburner M, Ball CA, Blake JA, Botstein D, Butler H, Cherry JM, et al. Gene ontology: tool for the unification of biology. The Gene Ontology Consortium. Nat Genet. 2000;25(1):25-9.

38. Lamesch P, Berardini TZ, Li D, Swarbreck D, Wilks C, Sasidharan R, et al. The Arabidopsis Information Resource (TAIR): improved gene annotation and new tools. Nucl Acids Res. 2012;40(Database issue):D1202-10.

39. Vanhaeren $H$, Inze D, Gonzalez N. Plant growth beyond limits. Trends Plant Sci. 2016;21(2):102-9.

40. Krajewski P, Chen D, Cwiek H, van Dijk AD, Fiorani F, Kersey P, et al. Towards recommendations for metadata and data handling in plant phenotyping. J Exp Bot. 2015;66(18):5417-27.

41. Miyoshi NS, Pinheiro DG, Silva WA Jr, Felipe JC. Computational framework to support integration of biomolecular and clinical data within a translational approach. BMC Bioinformatics. 2013;14:180.

\section{Submit your next manuscript to BioMed Central and we will help you at every step:}

- We accept pre-submission inquiries

- Our selector tool helps you to find the most relevant journal

- We provide round the clock customer support

- Convenient online submission

- Thorough peer review

- Inclusion in PubMed and all major indexing services

- Maximum visibility for your research

Submit your manuscript at www.biomedcentral.com/submit 